\title{
RESEARCH AND DEVELOPMENT OF DSP-BASED FACE RECOGNITION SYSTEM FOR ROBOTIC REHABILITATION NURSINGBEDS
}

\author{
Ashwini A. Patil ${ }^{1}$, Anita L. Devkar ${ }^{2}$, Asmita A. Pawar ${ }^{3}$ \\ ${ }^{1,2,3}$ Lecturer, IT Department, MCOE, Pune, Maharashtra, India
}

\begin{abstract}
This article describes the development of DSP as the core of the face recognition system, on the basis of understanding the background, significance and current research situation at home and abroad of face recognition issue, having a in-depth study to face detection, Image preprocessing, feature extraction face facial structure, facial expression feature extraction, classification and other issues during face recognition, and have achieved research and development of DSP-based face recognition system for robotic rehabilitation nursing beds. The system uses a fixed-point DSP TMS320DM642 as a central processing unit, with a strong processing performance, high flexibility and programmability.
\end{abstract}

Keywords: DSP; Face Detection; Face Recognition; Facial Expression Recognition *** $*$

\section{PREFACE}

The current situation of aging population more serious, the proportion of incapacitating and half disabling elderly people increased, the utilization rate of the robot rehabilitation nursing bed in the home and medical institutions have also increased significantly. In addition, the good and bad quality of care industry personnel, the vocational skills and responsibility nursing staff vary a lot, how to implement real-time monitoring of the patient's condition, avoiding unforeseen circumstances have become an important issue faced by families and caregivers. If it can add face recognition system in rehabilitation nursing robots bed, not only can provide the patient's real-time situation for the family or caregiver, norm professional ethics of care workers, to avoid abusing the elderly and other serious incidents occurred, but also to improve the productivity of medical institutions health care workers(Implement the centralized management and distributed control of multiple wards and multiple beds). Health care workers can monitor patients directly through the network in the computer terminals, health care workers immediately take appropriate medical measures after detecting an abnormal condition, , especially has an extremely important role for the care of critically ill patients. Additionally, it can provide remote medical care and reduce the costs of health care(Wherever important physiological information of the patient can be delivered to a remote medical center or home care expert through wireless communication in real time, accurate and fast and achieve automatic monitoring ).

Face Recognition, especially the computer technology of using analysis and comparison feature information of facial visual to identification Face recognition uses images or video streams containing human faces collected by a camera or camera , and automatically detecting and tracking human faces in the image, and then a series of related technology for the detected human face, often called the Face Recognition, Facial Recognition[1].

Face recognition based on the geometric characteristics is the oldest and popular recognition. The method is mainly using geometrical features of the facial , first positioning major organs of face images, such as the eyes, eyebrows, nose, mouth, face contour and chin, then select a set of features that can be characterized by the distance, the angle, the shape of the region and so on, then use the distance, ratio and other parameters as the identification of the characteristic information to deal with the face recognition. Face recognition system in rehabilitation nursing robot bed, efficiency and accuracy is the performance index that must be considered simultaneously. Based on this, a method of face recognition based on local matching is proposed. This method is based on the use of Haar classifier to realize the automatic detection of human face in dynamic video frames, extracting the $\mathrm{Hu}$ moments feature of facial features , using $\mathrm{Hu}$ moments feature collection to achieve the automatic recognition of human face and achieve real-time tracking[2].

\section{THE RESEARCH OF FACE RECOGNITION}

\section{SYSTEM}

Paragraph Face recognition system in Rehabilitation Nursing Robot bed is composed of face detection, face recognition, face tracking, facial expression recognition. Face recognition system flow chart is shown in Figure 1.The specific process of face recognition system as follows: After the camera is powered on, automatically reset to the preset position, and then began to cruise along the fixed route, to get a picture of a video from the surveillance video each 1s.To obtain the image of human face detection and location, to detect the face is to enter the face recognition process, or to continue to get the image.After the face is 
detected, the face image is pre processed, and the sample quality is improved without losing the main information of the sample. Then, the pre processed face images are segmented to segment the target area. Secondly, feature extraction is carried out on the separated target area. The extracted features are matched with the features of the human face image template database, and output the recognition results. When the user is identified as the database has been stored, the camera to stop cruising and start the face tracking. Then start to get a frame of video images every $2 \mathrm{~S}$ from the surveillance video, recognition of facial expressions. The feature extraction of facial expression image, and to match with the features of facial expression template database .The obtained expression results were combined with the user's vital sign parameters, compared with the data in DSP rules to determine the physiological needs of users, and drive the implementation of the corresponding operation machine. When an expression lasts for four seconds or more, it is judged to be a valid expression.

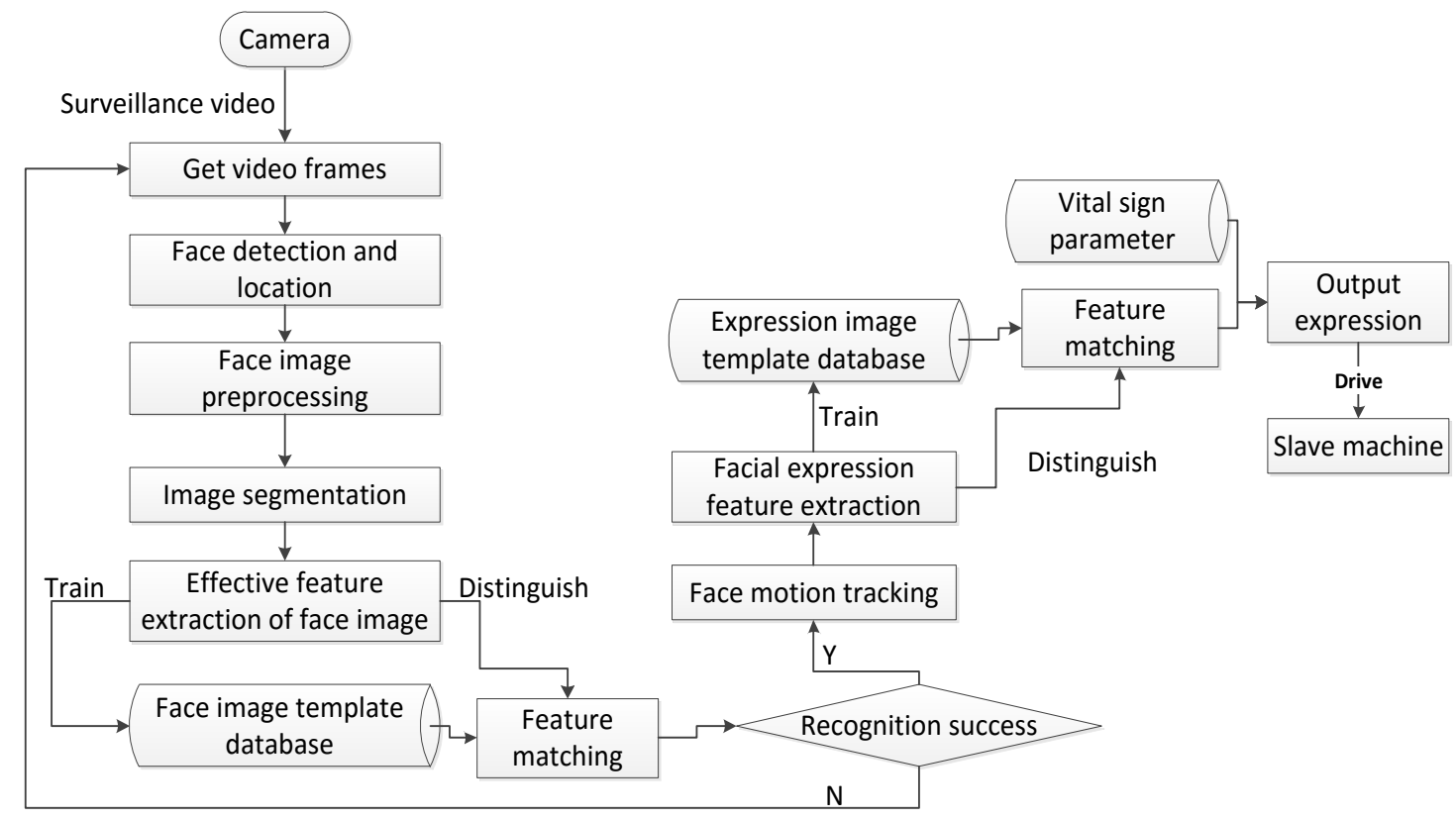

Fig-1 flow chart of face recognition system

\subsection{Face Detection}

Face detection uses face detection method of Haar classifier in the rehabilitation nursing bed robot. Compared with other face detection methods, Haar classifier is more effective for face detection in complex background, in the case of the same recognition rate of real-time better, is the most practical one of the most practical face detection method[3].As the base of face recognition, the accuracy of face detection plays an important role in face recognition. For the face detection in the rehabilitation nursing robot bed, the Haar classifier which trained by Adaboost is used to detect the human face in the dynamic video frame, to determine whether the video contains the face object, and finally give the information of location and size[4]. The specific detection process is shown in Figure 2(Figure 2 (b) in the red box marked for the human face of detection and localization.

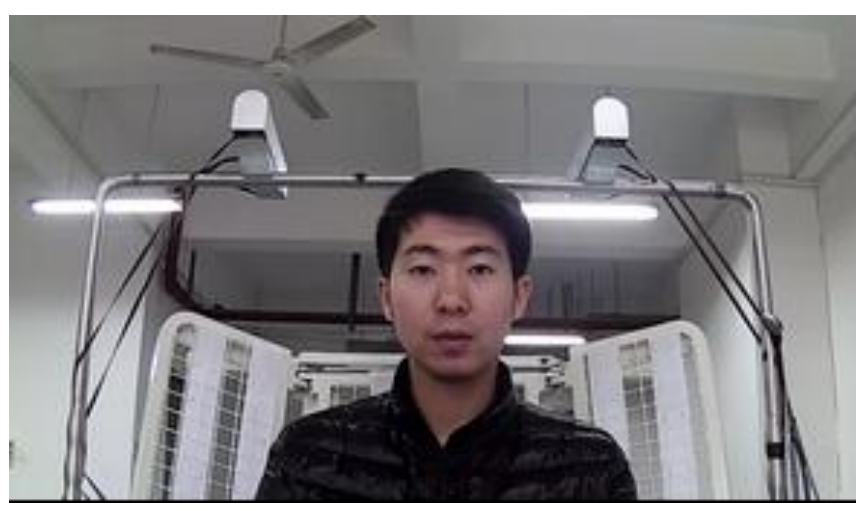

Fig -2: (a) a frame image in dynamic video

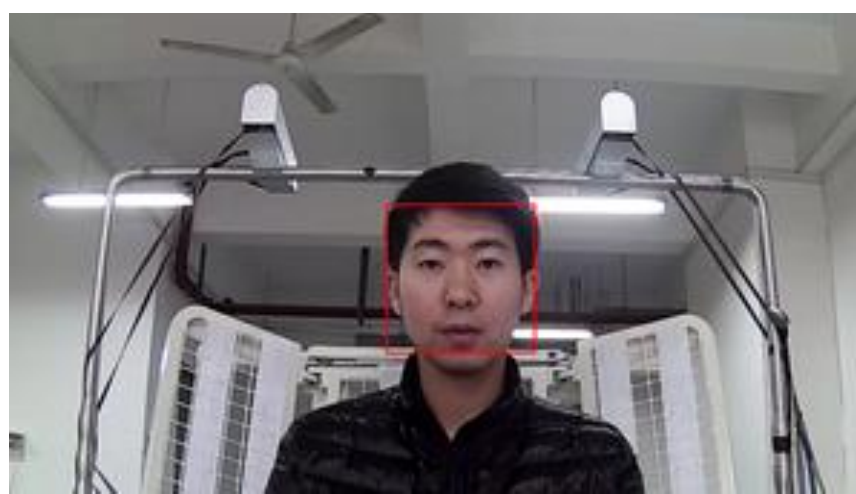

Fig -2: (b) face detection results 


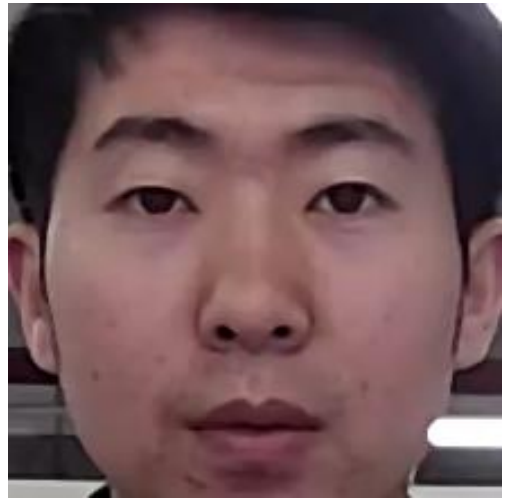

Fig -2: (c) face images

Fig-2 process and results of face detection

\subsection{Face Recognition}

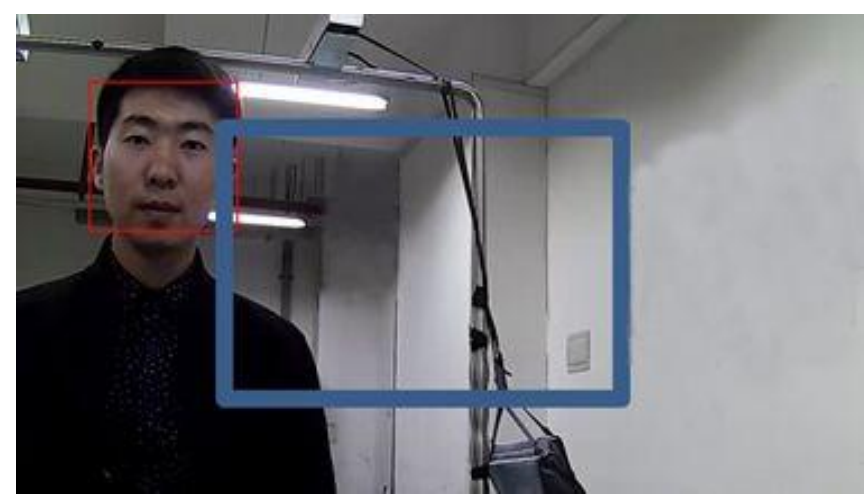

Fig-3.1 in the face outside the center of the image

The face recognition in the rehabilitation nursing robot bed is composed of three parts, which are image preprocessing, image segmentation and feature extraction. The image preprocessing is conducted before the training and test in the use of the sample, the sample image acquisition to the pretreatment, improving the sample quality. Bilateral filtering can remove acnode noise and keep image edge features, doesn't make the image produce significant fuzzy, is suitable for the treatment of facial images, so we have bilateral filtering on human faces of background segmentation. Image segmentation is to the human face image after median filtering, use Otsu threshold method, image binarization, respectively in the face image extracted the eyebrows, eyes and mouth regions of facial features[5]. Otsu method can save the image information features for subsequent feature extraction. Feature extraction is in face image segmentation in the eyebrows, eyes, mouth region, extract the feature points on the contour of the region.

The feature points meet the requirements that make maximum use the minimum feature points to characterize the shape information of the region contour[6].Using 8 feature points on both sides of the eyebrows contours shape representation of the eyebrows. Through these 16 points can distinguish different contours of the eyebrows. Because movement of the eye, right and left eyes of each with 5 points, 10 points to express the main shape of eyes[7].
The left corner and left the corners of the mouth are the benchmark points of using face reference coordinate system when the feature representation. This paper selects the following characteristics: as shown in Figure 3,the eyebrow has selected 8 feature points, the eye has selected 5 feature points, the mouth has selected 12 feature points, a total of 38 selected feature points to represent the face.

To determine the profile information of the corresponding characteristic parameters by matching the feature points on the contour and face recognition in the standard library and complete face recognition finally.

\subsection{Face Recognition}

Face tracking is the direction of movement of camera can automatically follow the face to move. After recognizing the corresponding user, judging whether the human face area is in the center of the image. The blue area is shown in the center area of the image. If the face is outside the center of the image ( Figure 4.1), the calculation of relative distance between the current position and the center region, regional approaches are used to automatically move the camera to make the final face in the image center area (Figure 3b)[8].

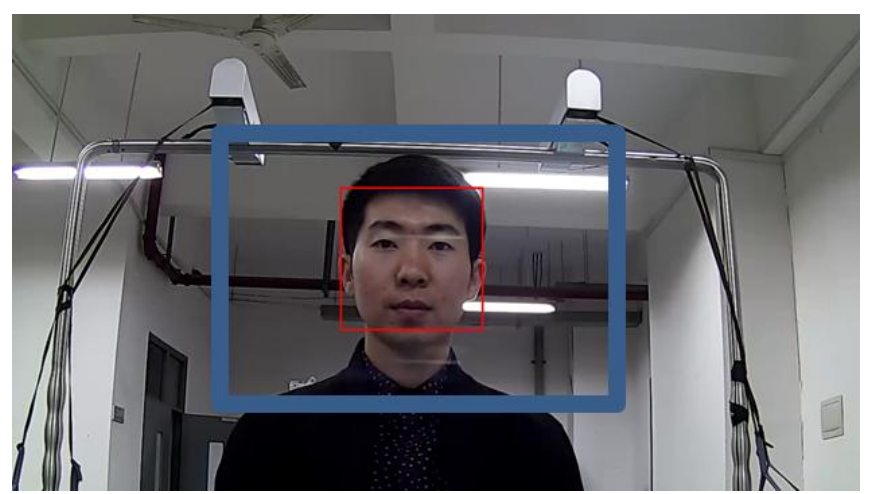

Fig. 3.2 results of region approximation

\subsection{Facial Expression Recognition}

Facial expressionrecognition is based on face recognition, calculating the offset of the characteristic value of the facial features in various facial expressions with respect to the standard face database.The storage standard face database is coordinates of the geometric features of the eyes, eyebrows and lips in no case of facial expression.Using The calculated facial features offset and real time four vital sign parameters (blood pressure, pulse, body temperature, respiration)as the former order of the rule base of the expert system is stored in the rule base. According to the medical staff on experience diagnosis of the disabled semi and disabled patients and field debugging experience, establishing the corresponding reasoning mechanism. According to the realtime measurement characteristic parameters of facial expression and vital signs parameters, reasoning and deduction based on facial expression recognition expert system knowledge database, get the judgment and decision, output patient's facial expression results, to control the lower machine driver, to meet the physiological needs of patients( Figure 3). 
Facial expression recognition is based on the face recognition,when the extraction of different expressions, offset of shape, size, position information of eyebrows, eyes and lips of facial features relative to standard expression(Figure 6).In the image below, the expression of surprise or anxiety compared to the normal state, changes in the external contour of eyes, eyebrows and lips are shapes and relative displacement. Through the camera to get the image and directly transfer to the DSP processing. The result of the treatment is matched with the expression in the standard database, then combined with vital signs parameters to judge the physiological needs of patients and execute the corresponding operation.
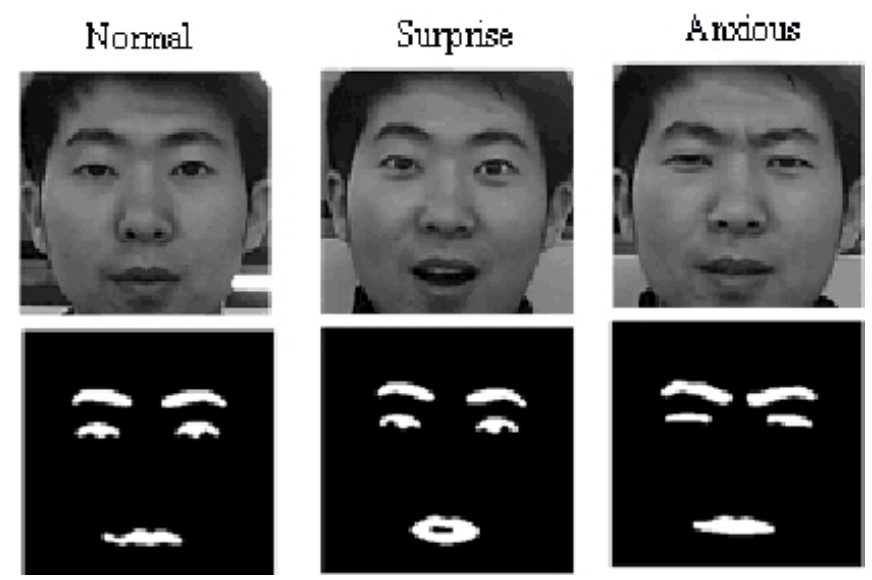

Fig-4 The relative changes of facial features in different expressions

\section{EXPERIMENTAL RESULTS AND ANALYSIS}

The 10 face recognition of each of the 10 test subjects were made by using the self-developed infrared network camera. There are 9 times failure, 2 times of which is caused by the face detection error. The specific identification results are shown in Table 2(The recognition time is the required time that a complete face detection and recognition process to complete).

Tab-1 results of face recognition

\begin{tabular}{|cccc|}
\hline $\begin{array}{l}\text { Total } \\
\text { number of } \\
\text { recognition }\end{array}$ & $\begin{array}{l}\text { Recognition } \\
\text { error times }\end{array}$ & $\begin{array}{l}\text { accuracy } \\
\text { rate/\% }\end{array}$ & $\begin{array}{l}\text { Recognition } \\
\text { time/s }\end{array}$ \\
\hline 100 & 9 & $91 \%$ & 4.3 \\
\hline
\end{tabular}

From table 1, the local matching face recognition method based on Hue moments can effectively recognize the human face in dynamic video frames, it has a high practical value.

\section{CONCLUSIONS}

Rehabilitation nursing robot bed adds intelligent system , especially the face recognition system can effectively solve the nursing problem which the aging of the population bring about. A face recognition system based on DSP is proposed in this paper. The system makes full use of the characteristics ability of the geometric features of facial features, based on the realization of automatic detection of human face in dynamic video frames using Haar classifier. The face image captured use $\mathrm{Hu}$ moments feature collection for automatic recognition and real-time tracking. The whole process is carried out in DSP, and the upper computer is not required.DSP gets the image through the serial port from the camera, recognition of expression is in DSP. The identification results combine with the vital signs parameters which vital signs measuring instrument transmitted. Both of them determine the physiological needs of patients. The result of the judgment is directly transmitted to the ARM control board through the serial port, which drives the slave computer to execute the corresponding operation. The method combines the expression recognition result, the vital sign parameter and the control system of the rehabilitation nursing robot bed. It improves furtherly the intelligence and practicality of the rehabilitation nursing robot bed.

\section{REFERENCES}

[1]. Yang G, Huang T S. Human face detection in complex background. Pattern Recognition[J], 1994, 27(1):53-63

[2]. Yuille A, Hallinan P and Cohen D. Feature extraction from faces using deformable templates. International Journal of Computer Vision, 1992, 8(2): 99-111.

[3]. Jolliffe I T. Principal Component Analysis. Springer0Verlag, New York, 1986.

[4]. Lin SH, Kung SY, Lin L J. Face recognition/detection by probabilistic decision-based neural network. IEEE Transactions on Neural networks, 1997, 8(1): 114-132.

[5]. Agarwal M, Agrawal H, Jain N, et al. Face recognition using principle component analysis, eigenface and neural network[C]. Signal Acquisition and Processing, 2010. ICSAP'IO. International Conferenceon. IEEE, 2010:310-314.

[6]. Yu Y, Li Z M. Improvement of Feature-based Face Recognition Algorithm by Elastic Graph Matching[J]. Computer Engineering, 2011, 5: 075.

[7]. Yang J, Zhang D, Frangi A F, et al. Two-dimensional PCA: a new approach to appearance-based face representation and recognition $[\mathrm{J}]$. Pattern Analysis and Machine Intelligence. IEEE Transactions on,2004, 26(1):131-137.

[8]. Yu Y, Li Z M. Improvement of Feature-based Face Recognition Algorithm by Elastic Graph Matching[J]. Computer Engineering, 2011, 5: 075.

\section{BIOGRAPHIES}

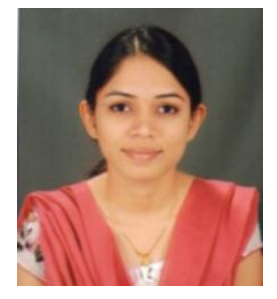

Ashwini A. Patil is a professor in the Information Technology Department, Modern College of Engineering, Pune, India. She received Master of Engineering (ME) degree from, Pune University. Her research interests are Software Engineering and Data Mining. 


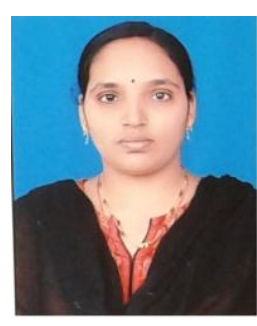

Anita L. Devkar is a lecturer in the Information Technology Department, Modern College of Engineering, Pune, India. She received Master of Technology (M Tech) degree from $\mathrm{COE}$, Pune. Her research interests are Information Retrieval, Data Mining and Cloud Computing

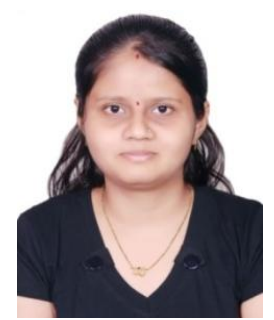

Asmita A. Pawar is a lecturer in the Information Technology Department, Modern College of Engineering, Pune ,India. She received Master of Engineering (ME) degree from North Maharashtra University, Jalgaon, India. Her research interests are Image Processing and Technologies. 